\title{
New Wolf-Rayet Galaxies with Detection of WC Stars
}

\author{
J.H. Huang ${ }^{1,2}$, Q.S. $\mathrm{Gu}^{1,2}$, L. $\mathrm{Ji}^{1,2}$, W.D. $\mathrm{Li}^{3}$, J.Y. Wei ${ }^{3}$ and W. Zheng ${ }^{4}$
}

Received —

\footnotetext{
${ }^{1}$ Department of Astronomy, Nanjing University, Nanjing, 210093, China

${ }^{2}$ United Lab for Optical Astronomy, The Chinese Academy of Sciences, 100080, China

${ }^{3}$ Beijing Astronomical Observatory, The Chinese Academy of Sciences, 100080, China

${ }^{4}$ Department of Physics and Astronomy, The Johns Hopkins University, Baltimore, MD 21218-2686
} 


\begin{abstract}
We report the discovery of two new Wolf-Rayet (WR) galaxies: Mrk 1039, and F08208+2816. Two broad WR bumps at $5808 \AA$ and $4650 \AA$ indicate the presence of WCE and WNL star population in all two sources. We also confirm the presence of WR features in Mrk 35, previously detected in a different position. The observed equivalent width of the WR bump at $4650 \AA$ and the derived number ratios of $\mathrm{WR} /(\mathrm{WR}+\mathrm{O})$ imply that star formation in these sources takes place in short burst duration. Comparisons with the recent models of WR populations in young starbursts with the observed EW(He II $\lambda 4686) / \mathrm{EW}(\mathrm{C}$ IV $\lambda 5808) / \mathrm{EW}(\mathrm{WRbump})$ and their relative intensities provide an indication that the stellar initial mass function in some WR galaxies might not be Salpeter-like. It is interesting to find that the luminous IRAS source, F08208+2816, has little dust reddening, probably because of the existence of a powerful superwind. By comparisons with other starbursts observed with the Hopkins Ultraviolet Telescope, F08208+2816 as a merging system renders a chance to study the contribution from young starbursts to the UV background radiation in universe.
\end{abstract}

Subject headings: galaxies: starburst — galaxies: individual: Mrk 35,Mrk 1039, F08208+2816 — stars: Wolf-Rayet 


\section{INTRODUCTION}

Wolf-Rayet (WR) galaxies are a subset of H II galaxies with broad emission lines that are characteristic of WR stars (Conti 1991). Outside the local universe, the broad feature usually detected in WR galaxies is the so-called WR bump, a blend of He II and other lines from C III, N III/N v. Among about 90 known WR galaxies (Schaerer 1997, private communication), thirteen show the broad C IV $\lambda 5808$ line, a strong feature from WC stars. Indeed, from the current knowledge of stellar evolution one would expect to detect WC stellar population in about $1 / 3$ of all WR galaxies with a metallicity of $(1 / 5-1) Z_{\odot}$ (Schaerer \& Vacca 1998, SV98 hereafter). The deficiency of such detection might be caused by the weakness of C IV $\lambda 5808$ emission, as compared with the WR bump. Alternatively, uncertainties in our knowledge of the evolution of massive stars (e.g. their mass-loss rates, Meynet 1995) could be responsible for such an apparent deficiency. For example, the recent discovery of WR stars in I Zw 18 (Izotov et al. 1997a, Legrand et al. 1997) seems to indicate enhanced mass-loss rates. These uncertainties also complicate derivations of the upper end of the initial mass function (IMF), which is a fundamental parameter in studies of WR galaxies (SV98; Leitherer 1996). For example Contini et al. (1995) report the observations of a new WR galaxy Mrk 712 which may require a flat IMF (Contini, Davoust, \& Considère 1995). Extending the sample of WR galaxies and studying both their WN and WC content is therefore very useful to constrain the evolution of massive stars and study the process of star formation in starbursts.

We report the discovery of two new WR galaxies from our systematic search for WR galaxies. One of them, Mrk 1039, was reported with a classification as H II galaxy, but the presence of broad emission lines in their spectra was not noticed (de Grijp et al. 1992). WR signatures were also found in Mrk 35 (Haro 3), previously studied by Steel et al. (1996). However, we observed this source in a different slit position, obtaining different WR features from those of Steel et al. In this paper, we present the observations and data reduction in $\S 2$, and discuss line measurements and the WR populations in these sources in $\S 3$. The implications from the comparisons between the observations and model predictions are discussed in $\S 4$.

\section{OBSERVATIONS}

Spectra of Mrk 35, Mrk 1039, and F08208+2816 were obtained under photometric conditions on 1997 January 10-11, February 6, November 21-23, and on 1998 February 28 at Beijing Astronomical Observatory (BAO) with a $2.16 \mathrm{~m}$ telescope. A grating with 300 lines $\mathrm{mm}^{-1}$ yields a spectral resolution of $11 \AA$ (FWHM) over the range of $3700-8000 \AA$. The total exposure time is $1200 \mathrm{sec}(600+600 \mathrm{sec})$ for Mrk $35,12600 \mathrm{sec}$ 
$(3 \times 3600+1800 \mathrm{sec})$ for Mrk 1039, and $10800 \mathrm{sec}(3 \times 3600 \mathrm{sec})$ for F08208+2816, respectively. The seeing was quite stable during the observations, at about $1 .^{\prime \prime} 5-2 .^{\prime \prime} 0$. A slit width of $2 .{ }^{\prime \prime} 5$ was taken for all three sources. Mrk 35 was observed with a position angle of $150^{\circ}$, different than the slit position used by Steel et al. (1996), whose observations we only became aware of recently.

The data were processed with standard IRAF procedures. All observed line fluxes have been corrected for reddening due to the dust in our Galaxy and within the sources themselves. We have followed the procedures to perform the absolute flux calibrations described in detail by Vacca \& Conti (1992). The underlying Balmer absorption of $\mathrm{EW} \sim 2 \AA$, which is the low-end of the predicted value for instantaneous burst at a metallicity $\mathrm{Z}<\mathrm{Z}_{\odot}$ (Olofsson 1995), was subtracted before correcting for intrinsic extinction. F08208+2816 is a unique source in this reduction, as discussed below.

The final de-reddened, rest-frame spectra are shown in Fig. 1a-1c, for Mrk 35, Mrk 1039, and F08208+2816, respectively. The enlarged spectra around $4650 \AA$ are also shown in the insets, in order to display the WR bump clearly. The broad C IV $\lambda 5808$ lines and the broad He II $\lambda 4686$ lines from WR stars are obviously present. The de-reddened line fluxes, relative to $\mathrm{H} \beta$, are given in Table 1 . For clarity, the WR line fluxes are listed separately in Table 2. Other measured properties are summarized in Table 3. The metallicities have been derived using McCall's algorithm with minor modifications (Pagel et al. 1992). McCall's algorithm is based on three-level-atom solutions, and hence is adequate at low and intermediate densities, with electron temperature and line ratio of $[\mathrm{O}$ III $](\lambda 4959+5007) / \lambda 4363$ as inputs.

Mrk 1039 has also been observed with medium-resolution, centered about the redshifted $4686 \AA$, with $2.16 \mathrm{~m}$ telescope at BAO on Oct 7-9, 1997 using 600 lines $\mathrm{mm}^{-1}$ grating, yielding resolution of $6.2 \AA$ as measured from the FWHM of the night sky lines. The total exposure time is $4 \times 3600$ sec. The spectra were dereddened using the internal reddening derived from low-resolution data listed in Table 3 . The final spectrum is illustrated in Fig. 3. The WR line fluxes and equivalent widths derived from medium resolution data are also listed in Table 2 , in parenthesis.

\section{WOLF-RAYET STARS IN Mrk 35, Mrk 1039, and F08208+2816}

Wolf-Rayet populations: According to the classification scheme of Smith, Shara, \& Moffat (1990b), a typical value of FWHM(C IV $\lambda 5808)$ for early-type WC stars is $60_{-10}^{+20} \AA$. The observed FWHM(C IV

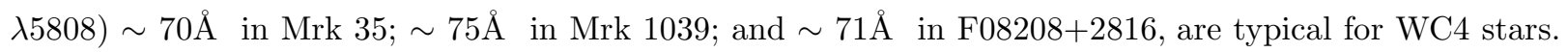


A substantial number of WO stars, i.e. comparable to the population of WCE stars, can be discarded in these objects, considering one critical criterion for the presence of WO stars is FWHM(C IV $\lambda 5808)$ $>90 \AA$, (Smith et al. 1990a, Smith \& Maeder 1991). Besides, we did not detect the O V $\lambda 5590$ line, a characteristic feature for WO1 \& 2 stars (Barlow \& Hummer 1982). This implies that, if WO stars exist in small quantities, they must be WO4 stars. There is no evidence for the presence of C III $\lambda 5696$ emission line in the spectra. We can therefore confidently exclude a major population of late type WC stars in Mrk 35, Mrk 1039, and F08208+2816.

Adopting the ionization subclass scheme for classifying WN subtypes (Smith et al. 1996), the early and late WN subtypes were demarcated by whether I(N v $\lambda 4606)$ is smaller (MN6 subtype) or larger than $0.5 \times \mathrm{I}(\mathrm{N}$ III $\lambda 4640)$ in peak fluxes. With this criterion, we found that the peak fluxes of $\mathrm{N} \mathrm{V} \lambda 4606$ in these three sources are below $0.5 \times \mathrm{I}(\mathrm{N}$ III $\lambda 4640)$, fitting well to late type WN stars. Thus, the WR populations in Mrk 35, Mrk 1039, and F08208+2816 are dominated by WNL and WCE stars.

Line measurements: The line measurement at the WR bump around $4650 \AA$ is a crucial step in the study of WR galaxies. A problem in line measurements is severe blending in this wavelength region. The WR bump around $4650 \AA$ may in general contain broad stellar emission lines, such as He II $\lambda 4686$, C IV $\lambda 4658, \mathrm{C}$ III $\lambda 4650, \mathrm{~N}$ III $\lambda \lambda 4634-4641, \mathrm{~N}$ V $\lambda 4619$, and $\mathrm{N}$ V $\lambda 4606$, but also narrow nebular emission lines including He II $\lambda 4686$, [Fe III] $\lambda 4658$, C III $\lambda 4650, \mathrm{~N}$ III $\lambda \lambda 4634-4641$, and [Ar IV] $\lambda 4711$. Our measurements were done using the "specfit" task in the STSDAS software by decomposing the WR bump into the six components (three nebular plus three stellar) between 4640 and $4711 \AA$ listed in Table 2 . Given the low spectral resolution and the moderate $\mathrm{S} / \mathrm{N}$ of $15-20$, the uncertainties may be larger than the formal values. This is illustrated by differences between measurements from low-resolution and medium-reslution observations for Mrk 1039, as we will discuss in $\S 4$.

\section{DISCUSSION}

WR/O number ratio: Vacca \& Conti (1992) have developed a method to derive the WR/O number ratio based on the flux of He II $\lambda 4686$ line relative to $\mathrm{H} \beta$. However, as discussed first by Conti (1993) and then by Schaerer (1996) and Schaerer \& Vacca (1998) in detail, some assumptions made by Vacca \& Conti (1992) in deriving WR/O ratios render this procedure uncertain. Among them, the evolutionary effects of $\mathrm{Q}_{\mathrm{o}}^{\mathrm{WR}}$, the average Lyman continuum luminosity per WR star, and the dominant factor $\eta_{\mathrm{o}}$, a conversion factor depending on age and other parameters, complicate the derivation of WR/O number ratios. In SV98, 
these two problems have been thoroughly investigated, supplying the evolution of $\mathrm{Q}_{\mathrm{o}}^{\mathrm{WR}}(\mathrm{t}, \mathrm{Z})$ and $\eta_{\mathrm{o}}(\mathrm{t}, \mathrm{Z})$ in replacing averaged $\mathrm{Q}_{\mathrm{o}}^{\mathrm{WR}}$ and $\eta_{\mathrm{o}}$. Furthermore, uncertainties in the mean He II $\lambda 4686$ line luminosity per WNL star are large, requiring further tests to verify its validity in deriving the number of WNL stars. Despite these errors, the estimate for the number of WNL stars using this mean luminosity is in good agreement with the number obtained by directly counting stars (c.f. SV98). Therefore the number ratios of $\mathrm{WR} / \mathrm{O}$, or $\mathrm{WC} /(\mathrm{WR}+\mathrm{O}), \mathrm{WNL} /(\mathrm{WR}+\mathrm{O})$ still help us understand the starburst phenomena. Adopting the mean He II $\lambda 4686 / \mathrm{C}$ IV $\lambda 5808$ line luminosity of $1.6 \times 10^{36} \mathrm{erg} \mathrm{s}^{-1} / 3.0 \times 10^{36} \mathrm{erg} \mathrm{s}^{-1}$ and appropriate values of $\mathrm{Q}_{\mathrm{o}}^{\mathrm{WR}}(\mathrm{t}, \mathrm{Z})$ and $\eta_{\mathrm{o}}(\mathrm{t}, \mathrm{Z})$ from the new model calculations of SV98, we have obtained the number of Wolf-Rayet and O stars, and the WR/(WR+O) number ratio thereafter in Mrk 35, Mrk 1039, and F08208+2816, given in Table 4.

From the comparisons of the relative number of WR stars with the model predictions (Cerviño \& Mas-Hesse 1994; Maeder \& Meynet 1994; Meynet 1995), we could see that the observed number ratio in these three sources are well above the predicted $\mathrm{WR} /(\mathrm{WR}+\mathrm{O})$ or $\mathrm{WR} / \mathrm{O}$ ratios for models with constant star formation regimes at metallicities of $(1 / 4-1 / 5) \mathrm{Z}_{\odot}$, which would be less than 0.01 , or 0.03 with high mass loss. Also the observed equivalent widths of the WR bump (see Table 2) are substantially larger than model predictions for constant star formation at a metallicity of $\sim 1 / 5 \mathrm{Z}_{\odot}, \mathrm{EW}(\mathrm{WRbump}) \sim 0.5 \AA$ (Cerviño \& Mas-Hesse 1994). Therefore, the event of star formation in Mrk 35, Mrk 1039, and F08208+2816 would be in short burst duration. The SV98 models for constant star formation (Version 2.32) give quite larger values for EW(WRbump) than those in Cerviño \& Mas-Hesse (1994). The above statement does, however, still hold.

In the instantaneous models, the burst age can be well indicated by the equivalent widths of $\mathrm{H} \beta$ (Copetti et al. 1986; Maeder \& Conti 1994; Cerviño \& Mas-Hesse 1994). The ages obtained from the latest synthesis models (SV98) are about 4.0Myr (Mrk 1039), 4.2Myr (Mrk 35), and 4.7Myr (F08208+2816).

Comparisons with new synthesis models: SV98 have constructed new synthesis models using up-to-date stellar evolution tracks and stellar atmospheres. The outputs of these models provide us with more detailed and self-consistent predictions for WR features. The comparisons of our observations with the SV98 model predictions are shown in Fig. 2, 4 and 5 for EW(He II $\lambda 4686) / \mathrm{EW}(\mathrm{WRbump}) / \mathrm{EW}(\mathrm{C}$ IV $\lambda 5808)$ and their relative intensities, respectively. The Figures show the relevant predictions for $Z=0.004$ and an IMF slope $\alpha=1$. (thick dashed line), $\alpha=2.35$ (Salpeter IMF, thick dot-dashed), and for $\mathrm{Z}=0.001$ (Salpeter IMF, thin dotted). All these lines are drawn with the model calculations Version 2.32 provided by Schaerer. Thick 
open symbols in these figures denote various observations: a square for Mrk 35, a triangle for Mrk 1039, and a circle for F08208+2816. Bars over these symbols show their uncertainties listed in Table 2, calculated following Kobulnicky \& Skillman (1996).

Fig. 2 shows that the observations of He II $\lambda 4686$ (line intensities and equivalent widths) in all objects are systematically larger than the model predictions with a Salpeter IMF, implying that a top-heavy IMF $(\alpha=1.0)$ might exist in these WR galaxies to favor the formation of WR stars. The large uncertainties of about 40-45\% in the measurements, however, make this conclusion vague (Schaerer 1997, private communication). Particularly, as most of our data are of low resolution, the strong broad emission features may completely dominate the wavelength region around $4686 \AA$. The measured line fluxes of nebular He II $\lambda 4686$ are probably underestimated, and the intensities of stellar components are most likely overestimated.

The influence of spectral resolution on the measured nebular and stellar lines at the $4650 \AA$ region can be demonstrated by comparisons of observed WR quantities derived from medium-resolution data, shown in Fig. 3 and listed in parentheses in Table 2, along with the values derived from low-resolution data of Mrk 1039. In fact, the line intensity of [Fe III] $\lambda 4658$ relative to $\mathrm{H} \beta=100$, obtained from medium-resolution data, is $0.9 \pm 0.4$, twice as large as that estimated with low-resolution data. The relative line intensity of $\mathrm{C}$ IV $\lambda 4658$ is reduced to $0.96 \pm 0.4$ accordingly, compared with $1.1 \pm 0.6$ derived from low resolution data. The nebular and stellar He II $\lambda 4686$ line intensities are 0.6/5.6 estimated from medium-resolution data, compared to $0.5 / 6.2$ given in Table 2 . Interestingly only [FeIII] is significantly changed between the low and medium resolution spectra. This situation, however, needs to be confirmed with higher resolution data.

With a spectral resolution higher than the one we used in 1997 October, Izotov et al. (1994, 1996, $1997 b$ ) found that the relative intensity of nebular He II $\lambda 4686$ is in the range of $\sim 1$ to $3 \%$. Our estimated intensity of nebular He II $\lambda 4686$ is about $1 \%$. It is possible that a larger value of I(nebular He II $\lambda 4686$ ) will be found in these sources with data of higher resolution, and the relevant stellar emission will not be significantly larger than the model values. Since the He II $\lambda 4686$ line is a dominate component in WRbump, the same arguments are applicable for comparisons between observed and model EW(WRbump), shown in Fig. 4.

C IV $\lambda 5808$ is a weak WR feature in WR galaxies, however, it is less affected by blending than He II $\lambda 4686$ line in WR bump. One could expect that comparisons between observed and model C IV $\lambda 5808$ lines would provide clearer trends. Fig $5 a$ illustrates comparisons for relative line intensities, and Fig. $5 b$ for $\mathrm{EW}(\mathrm{C}$ IV $\lambda 5808)$. It is interesting to note that the observed $\mathrm{EW}(\mathrm{C}$ IV $\lambda 5808)$ of Mrk 1039 can 
be matched within uncertainty by model predictions based on the Salpeter IMF, which is contradictory to the comparisons given in Fig. $2 b$ for this source. In the mean time, the relative line intensity, I(C IV ג5808)/I(H $\beta)$, of Mrk 1039 can be fitted by model calculations, within uncertainty, based on flat IMF. It should be pointed out that, at the burst age of 4-5 Myr for our three WR galaxies, the continuum emission from the gas is negligible (Leitherer \& Heckman 1995). The observed equivalent widths of WR features are thus insensitive to the gaseous radiation. It may, however, be reduced by underlying stellar population. On the other hand, the relative WR line intensities are less affected by such dilution effects, though unrealistic comparisons for line intensities could be caused by the mismatch of slit width with the nebular emission regions (see, e.g. Meynet 1995; SV98). Due to this possible "light loss" of nebular emission outside the slit, the relative intensities of $\mathrm{C}$ IV $\lambda 5808$ could be increased by such a factor.

To the first order, we might estimate the "loss" fraction of the Balmer emissions by an approach suggested by Mas-Hesse (1997, private communication). With a $\mathrm{H} \beta$ profile along the slit, we can estimate the fraction of $\mathrm{H} \beta$ emission that we have accumulated for study. After taking this "loss" effect of $\mathrm{H} \beta$ emission into consideration, the observed line intensities and the $\mathrm{EW}(\mathrm{C}$ IV $\lambda 5808)$ for the three WR galaxies are indicated by the thin, open symbols in Fig. 5. The same corrections have been made for observed values of He II $\lambda 4686$ line and the WR bump, shown also by thin, open symbols in Fig. 2 and 4 . The results obtained this way might indicate that the fitness to observed C IV $\lambda 5808$ in Mrk 1039 seems to move away from model predictions based on the Salpeter IMF, as compared with those before making correction on "loss" effect. For Mrk 35, however, the match is always held, before and after this correction, to the canonical models. F08208+2816 may be a source showing a clear trend of disparity with canonical models for C IV $\lambda$ 5808. The difference between the observed and the model predictions for this source, however, may not be as large as indicated in the figures. Because of the different approaches taken, as discussed in SV98, the duration of WC phase between the predictions of SV98 and Meynet (1995) are different, although by use of the same stellar models. WC phase in Meynet (1995) is longer than that in SV98. More realistic predictions on WC phase may be longer than that values we used for comparisons.

These comparisons with our low-resolution data tentatively suggest a deviation from the Salpeter IMF (1955) in some WR galaxies, which are worth studying at higher resolution. We are planning to do so, especially considering the fact that both He II $\lambda 4686$ and C IV $\lambda 5808$ are detected in these WR galaxies.

The number ratio of $\mathrm{WC} / \mathrm{WN}$ derived from these two features can provide critical tests on the mass-loss rates, a key input for stellar evolution models of massive stars (Maeder \& Meynet 1994, Meynet 
1995), which has also important consequences for population synthesis models. Indeed, different mass-loss rates can produce different distributions of WR stars among WC and WN subtypes. For example, the number ratio of WC/WNL $<1$ has been predicted by models of instantaneous starburst with standard mass loss rates at burst age of $4 \mathrm{Myr}$ for a metallicity of $\mathrm{Z}=0.004$, while $\mathrm{WC} / \mathrm{WNL}>1$ by models with high mass-loss rates (Meynet 1995). SV98 models are the ones with high mass-loss rates. The observed number ratios WC/WNL for Mrk 35/Mrk 1039/F08208+2816 are 0.33/0.34/0.42 at age of 3.9/3.6/4.4 Myr (newly derived ages corrected for the 'loss' of nebular emission as discussed above, also see Table 4), respectively. To be precise, the number of WNL stars should be derived from the luminosity of observed He II $\lambda 4686$ after subtraction of the contribution from WC stars to He II $\lambda 4686$ (de Mello et al. 1998). In the compilation of WR line luminosities in SV98, this contribution is included in 4650 blend. For WC4 stars, the He II $\lambda 4686$ from WC stars contributes $8-30 \%$ of the 4650 blend, based on the estimation of Smith et al. (1990a). The 'precise' number of WNL stars could be estimated from the luminosity of $\left(\mathrm{L}_{\mathrm{obs}}(\mathrm{He}\right.$ II $\left.\lambda 4686)-\mathrm{N}(\mathrm{WC}) \times f r a c \times 1.71 \times 3.0 \times 10^{36}\right)$, where frac denotes the fraction of 4650 blend being the contribution of He II $\lambda 4686$ from per WC star, and 1.71 is the mean flux ratio of 4650 blend with respect to C IV $\lambda 5808$ for WC4 star, adopting from Table 2 of SV98. The values listed in parentheses in the row 'WNL' of Table 4 are those 'precise' number of WNL stars, taking frac=19\%. The newly derived number ratios then become $0.42 / 0.43 / 0.57$, respectively, suggesting no need of high mass-loss rates for these three WR galaxies. The highest number ratio of WC/WNL, in the case of F08208+2816 and frac $=30 \%$, is 0.72 . To change high mass-loss rates to standard ones will significantly reduce the formation of WR stars and the duration of WR phase, as discussed by Meynet (1995), deteriorating the comparisons with our observations shown in Fig 2, 4 and 5. Due to a probable overestimation of line intensities in He II $\lambda 4686$ discussed above, more realistic WC/WNL number ratios will be derived from data of higher resolution, and the unsettled dilemma might be eased. We may find new constraints on models suitable for the new WR galaxies reported here.

It is worth mentioning the detection of He II $\lambda 5411$ (FWHM $\sim 24 \AA$ ) with $3 \sigma$ in Mrk 1039, the first detection of this broad line from WR stars outside the Local Group. The detection of this line might be related to the exceptionally strong He II $\lambda 4686$ in Mrk 1039. Compared to the EW(He II $\lambda 4686$ ) in Mrk 1039, those in Mrk 35 and F08208+2816 are about half as much (Fig. 2), and we have found no evidence of He II $\lambda 5411$ in these two sources.

F08208+2816: F08208+2816 is a luminous IRAS galaxy. Its infrared luminosity is L(8$1000 \mu m)=1.4 \times 10^{11} \mathrm{~L}_{\odot}$, following Sanders \& Mirabel (1996). If we take the Balmer decrements as 
a reddening tracer, then F08208+2816 has little internal reddening by dust. Indeed, the observed $\mathrm{F}(\mathrm{H} \alpha) / \mathrm{F}(\mathrm{H} \beta)$ ratio fits well, within uncertainty, to the theoretical $\mathrm{I}(\mathrm{H} \alpha) / \mathrm{I}(\mathrm{H} \beta)$ derived from Case B recombination. Also, the correction for the underlying Balmer absorption for this source would not need to be greater than $0.25 \AA$.

Fig. 6 shows the field centred about F08208+2816, extracted from the Digitized Sky Survey. The WR features are detected in Source 2. Source 4 is a foreground star. Source 3 is too faint for the $2.16 \mathrm{~m}$ telescope at BAO. Source 1 is faint, and shows properties of a H II galaxy with the same redshift as Source 2. Apparently, F08208+2816 is a merging system. It is therefore another example of a luminous IRAS galaxy (LIRG) indicating the merging process as a triggering mechanism for starburst (Sanders et al. 1988). And it is the second LIRG with detection of WR stellar population or young massive O stars, providing direct evidence for the connection between starburst and very powerful far-infrared emission. The other two sources are IRAS 01003-2238 (a WR galaxy, Armus, Heckman \& Miley 1988), and IRAS 08339+6517 (Gonzalez-Delgado et al. 1998). The luminosity of L(WRbump) $(\mathrm{F} 08208+2816)=3.8 \times 10^{40} \mathrm{ergs} \mathrm{s}^{-1}$, about a decade lower than that in IRAS 01003-2238 (Armus et al. 1988). Interestingly, F08208+2816 is the only source in these three LIRG that shows essentially zero internal reddening, as derived from the Balmer decrements.

It is possible that some of the dust clouds responsible for infrared emission could be located close to the H $\alpha$-emitting gas, as suggested by Maíz-Apellániz et al. (1998) for their observations of NGC4214. The scattering of $\mathrm{H} \alpha$ photons would reduce the Balmer ratio. Alternatively, the stellar winds from massive stars could expel the interstellar medium and the region we observed might then be density bounded instead of ionization bounded.

In view of the existence of a large number of young massive stars in Source 2 of F08208+2816 $\left(>2 \times 10^{4}\right.$ WR stars, or $\sim 1.4 \times 10^{5}$ massive O stars) a powerful superwind could be expected in F08208+2816. Therefore the interstellar medium in Source 2 has probably been blown off. The region we studied is likely not ionization bounded, implying that a large fraction of the Lyman-continuum photons of $1.4 \times 10^{54} / \mathrm{sec}$ might escape from Source 2 of F08208+2816. Indeed, IRAS $08339+6517$ is a source with about the same infrared luminosity as F08208+2816, its internal reddening derived from the Balmer decrements is 0.55 mag (Leitherer et al. 1995). The escape fraction of Lyman continuum photons, estimated from the observed flux at about $900 \AA$ with the Hopkins Ultraviolet Telescope (HUT), is < 4.1\% (Hurwitz et al. 1997). Another starburst galaxy observed with HUT, Mrk 66, shows same zero internal reddening as Source 2 
of F08208+2816, but with lower infrared luminosity, about five times less than that of F08208+2816. It is interesting to see that the upper limit of escape fraction of Lyman photons from Mrk 66 is at least $43 \%$ (cf. Hurwitz et al. 1997). Based on these new results, F08208+2816 might be a promising source for understanding the contribution from young starbursts to the UV background radiation, especially considering that merging systems should have been more frequent in the early universe.

We would like to thank an anonymous referee for his thoughtful and instructive comments on our paper. A substantial revision was made following his very detailed suggestions. We are grateful to D. Kunth, M. Mas-Hesse and F. Legrand for helpful discussions and suggestions on an earlier version of the manuscript. We also thank D. Schaerer for providing us with SV98 models prior to publication, and for his critical comments on our later version, which strongly deepened our analyses. The BAO staffs are thanked for their assistance during the observations. This work is supported by a grant from the NSF of China, and a grant from the Ascent Project of the State Science Commission of China. 


\section{REFERENCES}

Armus, L., Heckman, T.M., \& Miley, G.K. 1988, ApJ, 326, L45

Barlow, M.J. \& Hummer, D.G. 1982, in Wolf-Rayet Stars: Observations, Physics, Evolution, ed. C. W. H. de Loore, A. J. Wills, (Reidel: Dordrecht), 387

Cerviño, M., \& Mas-Hesse, J.M. 1994, A\&A, 284, 749

Conti, P.S. 1991, ApJ, 377, 115

Conti, P.S. 1993, in Massive Stars: Their lives in the interstellar medium, ASP Conf. Series 35 , p.449

Contini, T., Davoust, E., \& Considère, S. 1995, A\&A, 303, 440

Copetti, M.V.F., Pastoriza, M.G., \& Dottori, H.A. 1986, A\&A, 156, 111

de Grijp, M.H.K., Keel W.C., Miley G.K., Goudfrooij P., \& Lub, J. 1992, A\&AS, 96, 389

de Mello, D.F., Schaerer, D., Heldmann, J., \& Leitherer, C. 1998, ApJ, in press.

González Delgado, R.M., Leitherer, C., Heckman, T., Lowenthal, J.D., Ferguson, H.C., \& Robert, C. 1998, ApJ, 495, 698

Hurwitz, M., Jelinsky, P., \& Van Dyke Dixon, W. 1997, ApJ, 481, L31

Izotov, Y.I., Dyak,A.B., Chaffe, F.H., Foltz, C.B., Kniazev, A.Y., \& Lipovetsky, V.A. 1996, ApJ, 458, 524

Izotov, Y.I., Foltz, C.B., Green, R.F., Guseva, N.G., \& Thuan, T.X. 1997a, ApJL, 487, 37

Izotov, Y.I., Thuan, T.X., \& Lipovetsky, V.A. 1994, ApJ, 435, 647 1997b, ApJS, 108, 1

Kobulnicky, H.A., \& Skillman, E.D. 1996, ApJ, 471, 211

Legrand, F., Kunth, D., Roy, J.-R., Mas-Hesse, J.M., \& Walsh, J.R. 1997, A\&A, 326, L17

Leitherer, C. 1996, STScI preprint No. 1175

Leitherer, C., Ferguson, H.C., Heckman, T.M., \& Lowenthal, J.D. 1995, ApJ, 454, L19 
Leitherer, C., \& Heckman, T.M. 1995, ApJS, 96, 9

Maeder, A., \& Conti, P.S. 1994, ARAA, 32, 227

Maeder, A., \& Meynet, G. 1994, A\&A, 287, 803

Maíz-Apellániz, J., Mas-Hesse, J.M., Muñoz-Tuñön, C., Vílchez, J.M., \& Castañeda, H.O. 1998, A\&A, 329, 409

Meynet, G. 1995, A\&A, 298, 767

Olofsson, K. 1995, A\&AS, 111, 57

Pagel, B.E.J., Simonson, E.A., Terlevich, R.J., \& Edmunds, M.G. 1992, MNRAS, 255, 325

Salpeter, E.E. 1955, ApJ, 121, 161

Sanders, D.B., \& Mirabel, I.F. 1996, ARAA, 34, 749

Sanders, D.B., et al. 1988, ApJ, 325, 74

Schaerer, D. 1996, ApJ, 467, L17

Schaerer, D., \& Vacca, W.D. 1998, ApJ, 497, 618 (SV98)

Schaerer, D., Contini, T., Kunth, D., \& Meynet, G. 1997, ApJ, 481, L75

Smith, L.F., \& Maeder, A. 1991, A\&A, 241, 77

Smith, L.F., Shara, M.M., \& Moffat, A.F.J. 1990a, ApJ, 348, 471 1990b, ApJ, 358, 229 1996, MNRAS, 281, 163

Steel, S.J., Smith, N., Metcalfe, L, Rabbette, M., \& McBreen, B. 1996, A\&A, 311, 721

Vacca, W.D., \& Conti, P.S. 1992, ApJ, 401, 543 
Table 1. Properties of the Spectral Lines

\begin{tabular}{|c|c|c|c|c|}
\hline \multirow{2}{*}{$\begin{array}{l}\lambda_{0} \\
(\AA)\end{array}$} & \multirow[t]{2}{*}{ Ion } & \multicolumn{3}{|c|}{ Intensity $^{\mathrm{a}}$} \\
\hline & & Mrk 35 & Mrk 1039 & F08208+2816 \\
\hline 3727 & {$\left[\begin{array}{ll}\mathrm{O} & \mathrm{II}\end{array}\right]$} & 155.0 & 98.0 & 151.2 \\
\hline 3869 & {$[\mathrm{Ne}$ III $]+\mathrm{He} \mathrm{I}$} & 22.8 & 20.3 & 29.6 \\
\hline 3967 & $\mathrm{H} \epsilon+[\mathrm{Ne} \quad \mathrm{III}]$ & 26.9 & 18.8 & 18.5 \\
\hline 4101 & $\mathrm{H} \delta$ & 25.1 & 14.7 & 20.9 \\
\hline 4340 & $\mathrm{H} \gamma$ & 46.0 & 35.3 & 42.8 \\
\hline 4363 & {$[\mathrm{O}$ III $]$} & 2.3 & 4.3 & 3.5 \\
\hline 4471 & $\mathrm{HeI}$ & 4.3 & 4.3 & 3.5 \\
\hline 4861 & $\mathrm{H} \beta$ & 100.0 & 100.0 & 100.0 \\
\hline 4959 & {$[\mathrm{O}$ III $]$} & 130.1 & 158.2 & 134.1 \\
\hline 5007 & [O III $]$ & 396.0 & 475.9 & 404.5 \\
\hline 5200 & {$[\mathrm{~N}$ II $]$} & 0.6 & 1.8 & 2.3 \\
\hline 5876 & He I & 13.1 & 15.1 & 13.2 \\
\hline 6304 & {$[\mathrm{O} \mathrm{I}]+[\mathrm{S} \mathrm{III}]$} & 4.1 & 6.0 & 7.1 \\
\hline 6563 & $\mathrm{H} \alpha$ & 286.6 & 282.9 & 282.0 \\
\hline 6584 & {$[\mathrm{~N}$ II $]$} & 16.9 & 14.5 & 33.6 \\
\hline 6716 & [S II] & 13.9 & 16.2 & 24.7 \\
\hline 6731 & {$[\mathrm{~S}$ II $]$} & 13.9 & 13.4 & 19.2 \\
\hline
\end{tabular}

${ }^{\text {a }}$ De-reddened line fluxes relative to $\mathrm{I}(\mathrm{H} \beta)=100$ 
Table 2. Wolf-Rayet Features

\begin{tabular}{|c|c|c|c|c|}
\hline \multirow{2}{*}{$\begin{array}{l}\lambda_{0} \\
(\AA)\end{array}$} & \multirow[t]{2}{*}{ Ion } & \multicolumn{3}{|c|}{ Intensity $^{\mathrm{a}}$} \\
\hline & & Mrk 35 & Mrk 1039 & F08208+2816 \\
\hline \multirow[t]{2}{*}{4640} & N III & $1.0 \pm 0.6$ & $0.7 \pm 0.4$ & $1.1 \pm 0.7$ \\
\hline & & & $(0.5 \pm 0.3)^{\mathrm{b}}$ & \\
\hline \multirow[t]{2}{*}{$4658^{n}$} & [Fe III $]$ & $0.2 \pm 0.1$ & $0.4 \pm 0.2$ & $0.8 \pm 0.5$ \\
\hline & & & $(0.9 \pm 0.4)$ & \\
\hline \multirow[t]{2}{*}{$4658^{\mathrm{s}}$} & C IV & $1.8 \pm 1.0$ & $1.1 \pm 0.6$ & $1.2 \pm 0.7$ \\
\hline & & & $(0.96 \pm 0.4)$ & \\
\hline \multirow[t]{2}{*}{$4686^{\mathrm{n}}$} & He II & $0.2 \pm 0.1$ & $0.5 \pm 0.3$ & .. \\
\hline & & & $(0.6 \pm 0.3)$ & \\
\hline \multirow[t]{2}{*}{$4686^{\mathrm{s}}$} & He II & $4.0 \pm 2.2$ & $6.2 \pm 2.5$ & $5.6 \pm 3.0$ \\
\hline & & & $(5.6 \pm 2.2)$ & \\
\hline \multirow[t]{2}{*}{4711} & [Ar IV] & $0.6 \pm 0.4$ & $0.7 \pm 0.4$ & $1.0 \pm 0.6$ \\
\hline & & & $(0.5 \pm 0.3)$ & \\
\hline 5411 & He II & $\ldots$ & $1.0 \pm 0.2$ & $\ldots$ \\
\hline 5808 & C IV & $2.5 \pm 0.8$ & $3.9 \pm 1.2$ & $4.5 \pm 1.7$ \\
\hline \multirow{2}{*}{\multicolumn{2}{|c|}{ EW(He II4686) }} & $6.0 \pm 3.3$ & $10.4 \pm 4.2$ & $4.5 \pm 2.5$ \\
\hline & & & $(9.9 \pm 3.9)$ & \\
\hline \multicolumn{2}{|c|}{ EW(C IV5808) } & $5.3 \pm 1.7$ & $7.7 \pm 2.3$ & $5.3 \pm 1.7$ \\
\hline \multirow{2}{*}{\multicolumn{2}{|c|}{ EW(WRbump) }} & $9.8 \pm 5.5$ & $14.8 \pm 6.7$ & $6.4 \pm 3.6$ \\
\hline & & & $(12.6 \pm 5.6)$ & \\
\hline
\end{tabular}

${ }^{\text {a }}$ De-reddened line fluxes relative to $\mathrm{I}(\mathrm{H} \beta)=100$

${ }^{\mathrm{b}}$ The values listed in parenthesis are those obtained from medium resolution data

${ }^{\mathrm{n}}$ Nebular, narrow emission lines

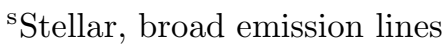


Table 3. Properties of $\mathrm{H} \beta$ and Reddening

\begin{tabular}{lrrr}
\hline \hline & & & \\
& Mrk 35 & Mrk 1039 & F08208+2816 \\
\hline $\mathrm{z}$ & 0.00327 & 0.00656 & 0.0472 \\
$\mathrm{EW}(\mathrm{H} \beta)(\AA)$ & 145.7 & 174.8 & 80.9 \\
$\mathrm{I}(\mathrm{H} \beta)\left(10^{-13} \mathrm{ergs} \mathrm{s}^{-1} \mathrm{~cm}^{-2}\right)$ & 6.17 & 6.36 & 1.14 \\
{$[\mathrm{O} / \mathrm{H}]$} & 8.32 & 8.17 & 8.15 \\
$\mathrm{E}(\mathrm{B}-\mathrm{V})$ & 0.112 & 0.469 & $\sim 0$ \\
$\mathrm{Distance}\left(\mathrm{Mpc}^{\mathrm{a}}\right.$ & 13.1 & 26.2 & 188.8 \\
\hline & & &
\end{tabular}


Table 4. $\mathrm{WR} /(\mathrm{WR}+\mathrm{O})$ number ratios

\begin{tabular}{lrrr}
\hline \hline & & & \\
& Mrk 35 & Mrk 1039 & F08208+2816 \\
\hline WC & 104 & 687 & 7213 \\
WNL & 313 & 2018 & $1.695 \times 10^{4}$ \\
& $(250)^{\mathrm{a}}$ & $(1602)^{\mathrm{a}}$ & $\left(1.258 \times 10^{4}\right)^{\mathrm{a}}$ \\
$\mathrm{O}$ & 3620 & 9720 & $1.895 \times 10^{5}$ \\
& $(2795)$ & $(6430)$ & $\left(1.419 \times 10^{5}\right)$ \\
$\mathrm{WC} /(\mathrm{WR}+\mathrm{O})$ & 0.026 & 0.055 & 0.034 \\
& $(0.033)$ & $(0.079)$ & $(0.045)$ \\
$\mathrm{WNL} /(\mathrm{WR}+\mathrm{O})$ & 0.077 & 0.16 & 0.079 \\
& $(0.079)$ & $(0.18)$ & $(0.078)$ \\
$\mathrm{WR} /(\mathrm{WR}+\mathrm{O})$ & 0.10 & 0.22 & 0.11 \\
& $(0.11)$ & $(0.26)$ & $(0.12)$ \\
$\mathrm{burst}$ age $(\mathrm{Myr})$ & 4.2 & 4.0 & 4.7 \\
& $(3.9)^{\mathrm{b}}$ & $(3.6)^{\mathrm{b}}$ & $(4.4)^{\mathrm{b}}$ \\
\hline & & &
\end{tabular}

${ }^{a}$ number of WNL stars after subtracting the contribution of WC stars to $4686 \AA$ line. The corrected new values of $\mathrm{O}$ stars and the number ratios are all listed in parentheses thereafter.

bage corrected for "light loss" of nebular emission 
Figure captions:

Figure 1a. Spectrum of Mrk 35 with low resolution, where two WR bumps around $4650 \AA$ and $5808 \AA$ are marked. The inset shows enlargement on the WR bumps around $4600 \AA$, where the broad He II $\lambda 4686$ line from Wolf-Rayet stars is positively detected.

Figure 1b. Same as Fig 1a, but for Mrk 1039.

Figure 1c. Same as Fig 1a, but for F08208+2816.

Figure 2. Comparisons between the predicted and observed quantities of stellar He II $\lambda 4686$ line for $\mathrm{Z}=0.004$ (dotted-dashed), 0.001 (thin dotted) based on Salpeter IMF, and $\mathrm{Z}=0.004$ (thick dashed) based on a very flat IMF $(\alpha=1.0)$, a) relative line intensities; b) equivalent widths. Observed data are denoted by thick open symbols: square for Mrk 35; triangle for Mrk 1039; and circle for F08208+2816. See the text for the thin open symbols.

Figure 3. Spectrum of Mrk 1039 with medium resolution taken in the blue wavelength band. The inset shows enlargement on the WR bump as those in Fig 1.

Figure 4. Comparisons between the predicted and observed equivalent widths of WR bump. Same notations used as those in Fig 1.

Figure 5. Same as Fig 1, but for C IV $\lambda 5808$ line.

Figure 6 . Field centered about F08208+2816, in $4^{\prime} \times 4^{\prime}$. North is up, and east is to the left. Sources $1-3$ are three components in F08208+2816 system. Source 2 is where WCE and WNL stars are detected. Source 4 is a foreground star. 


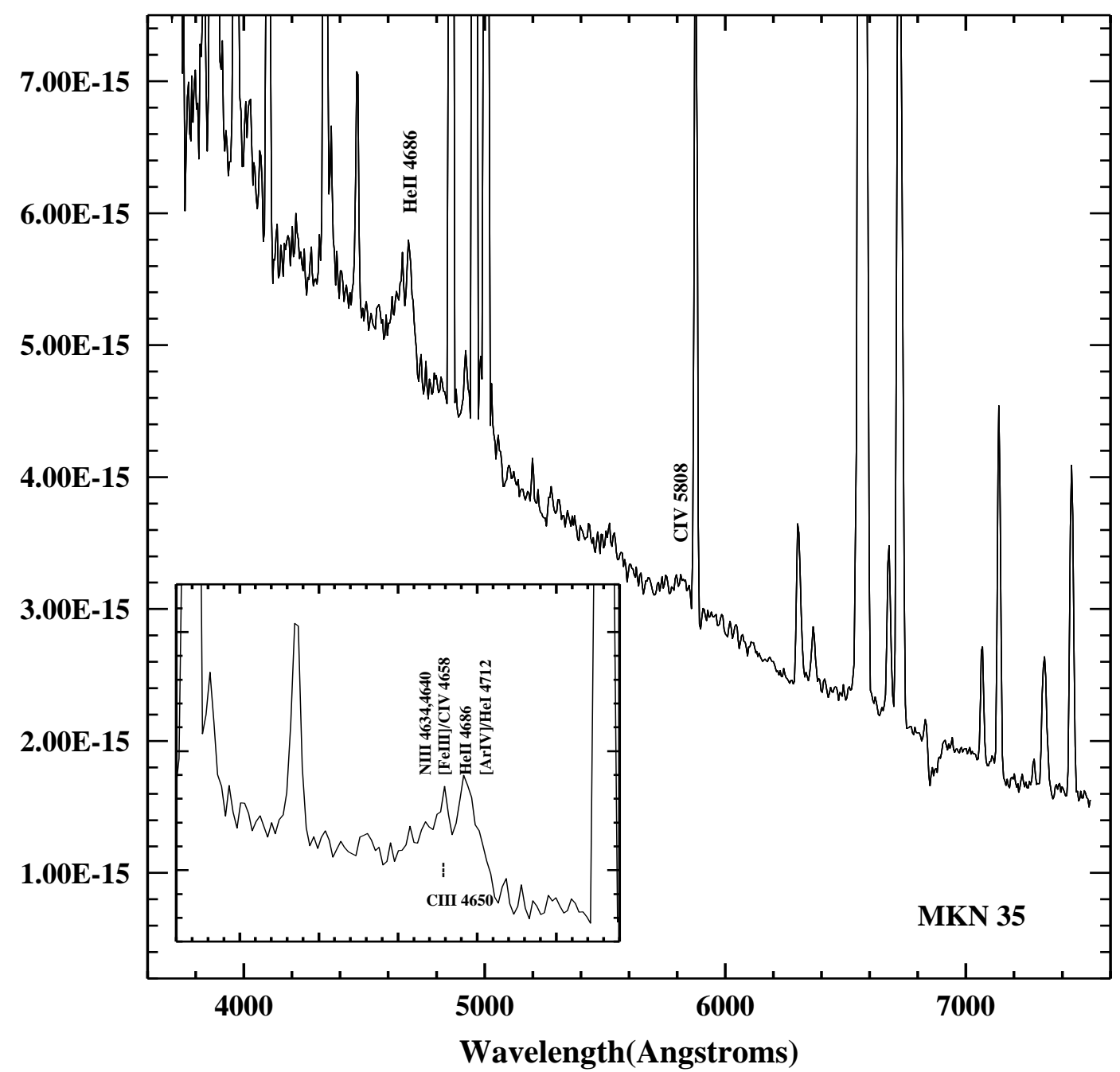




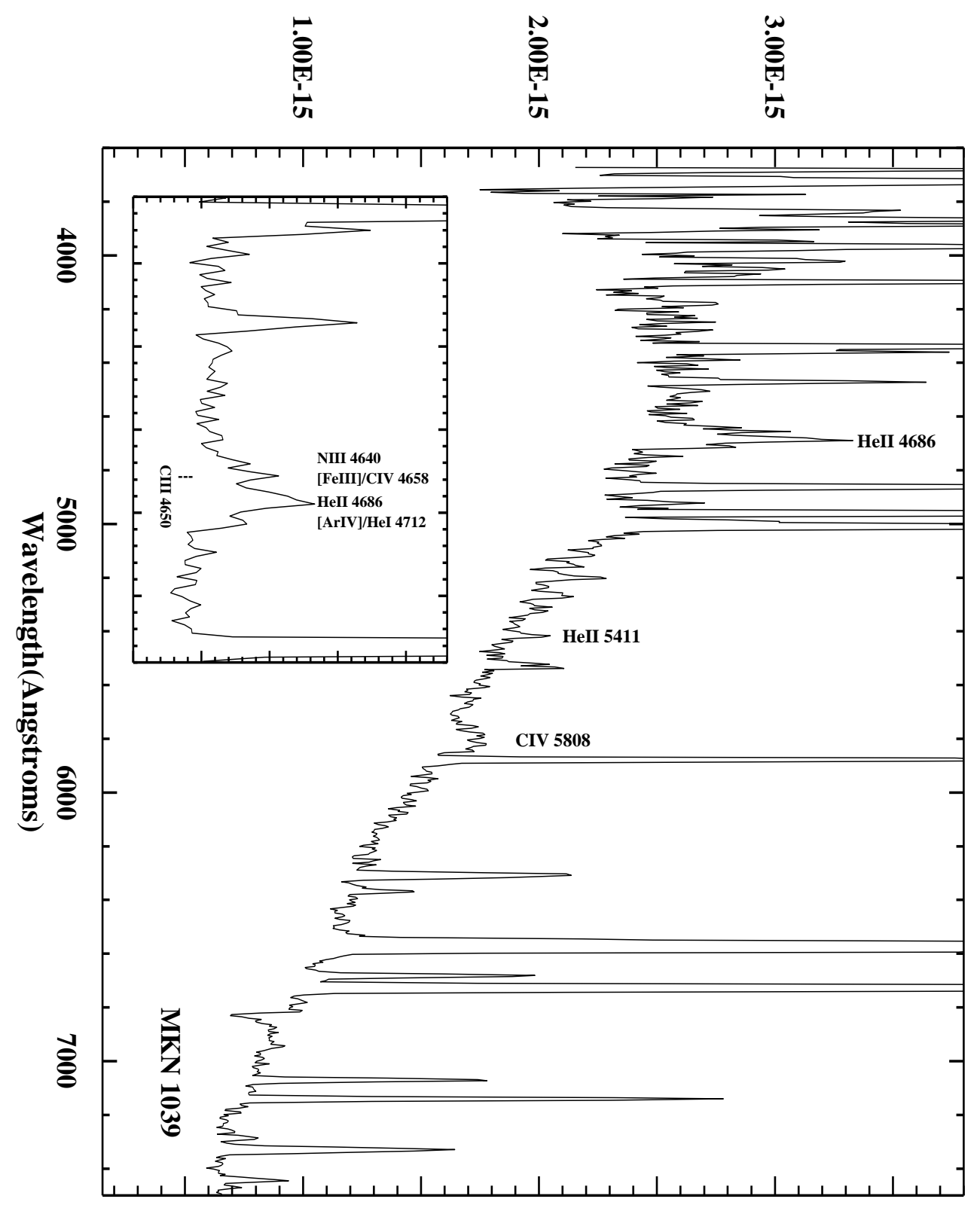




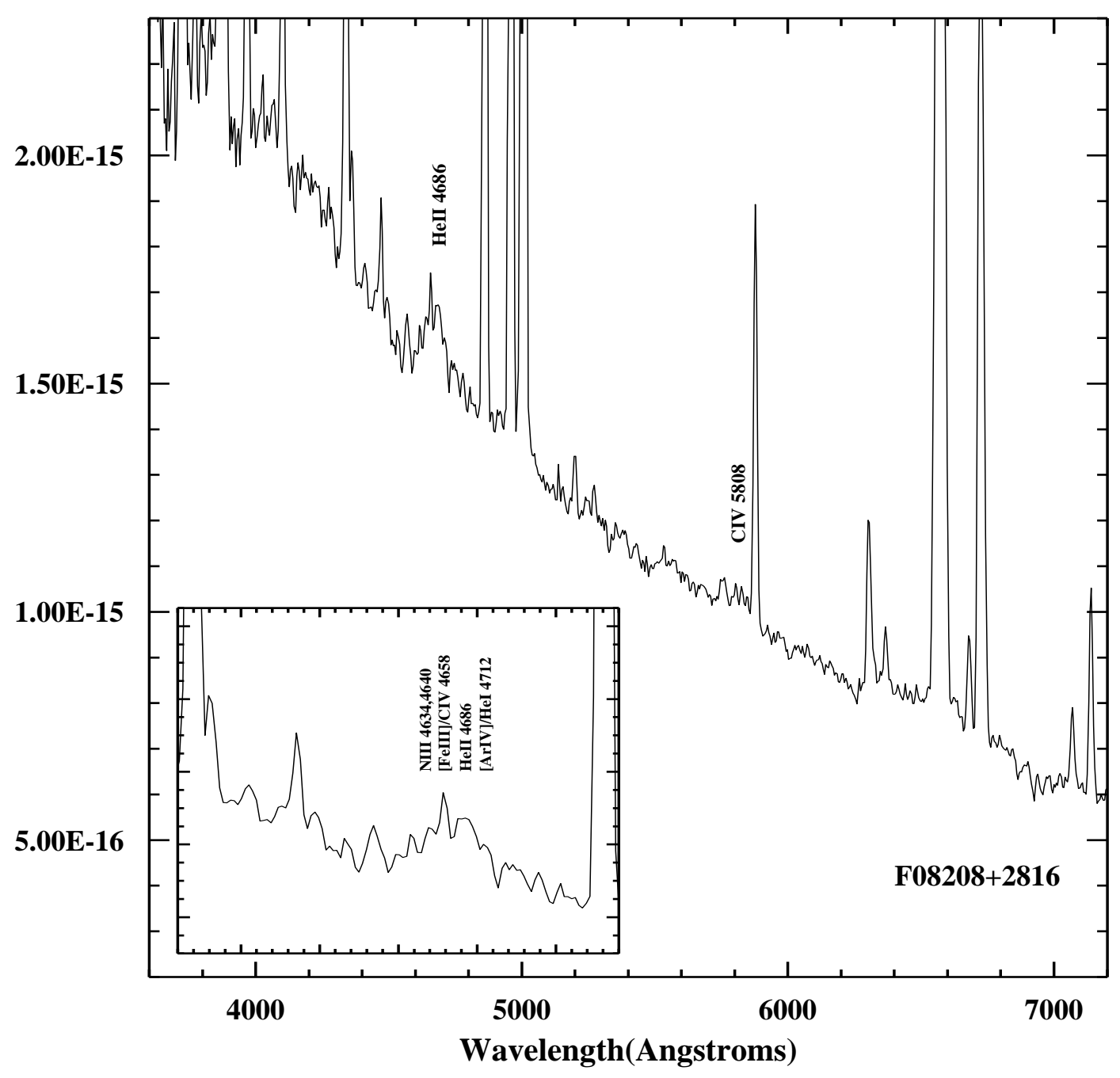




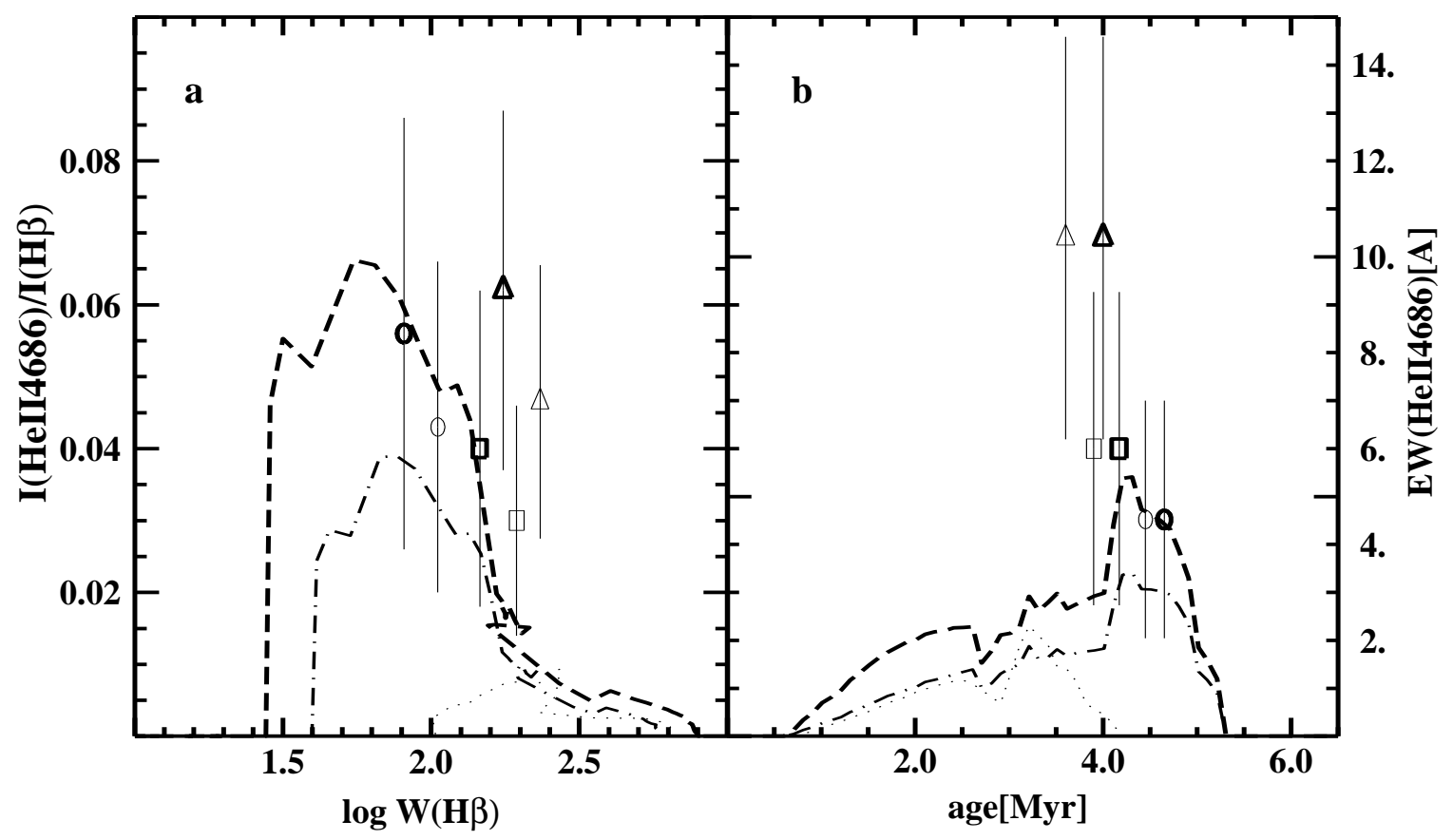




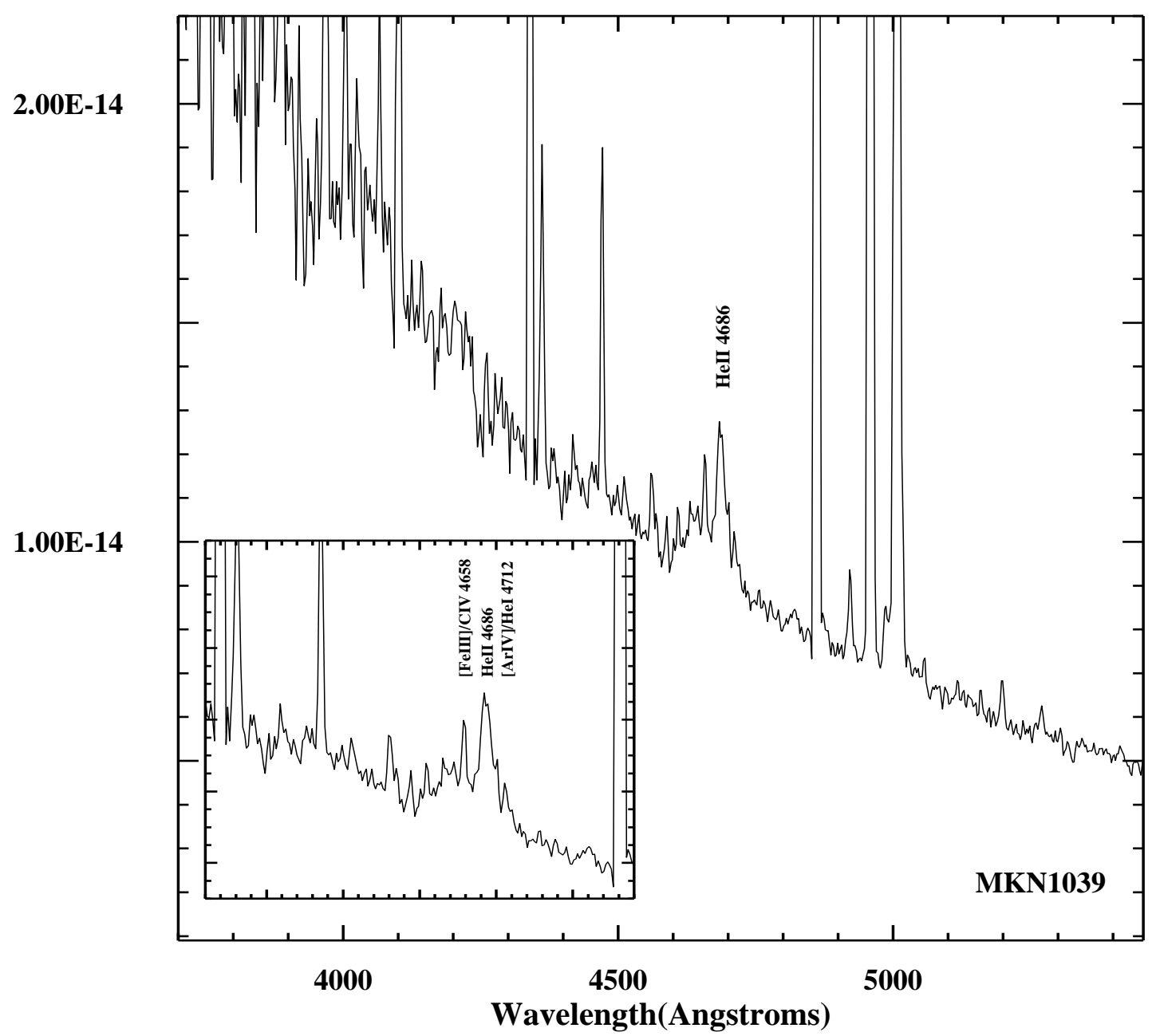




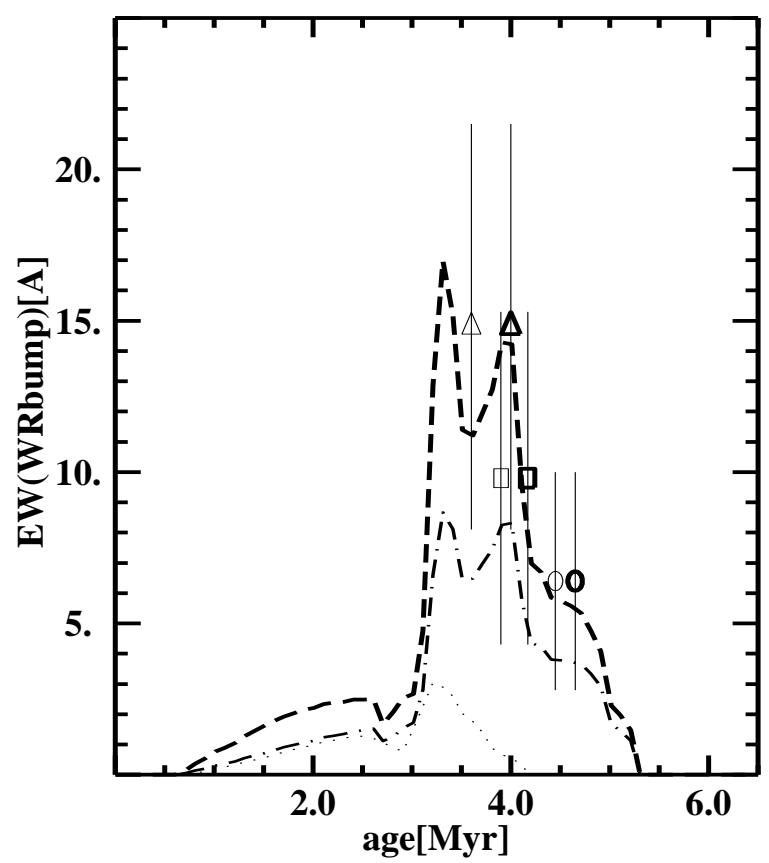




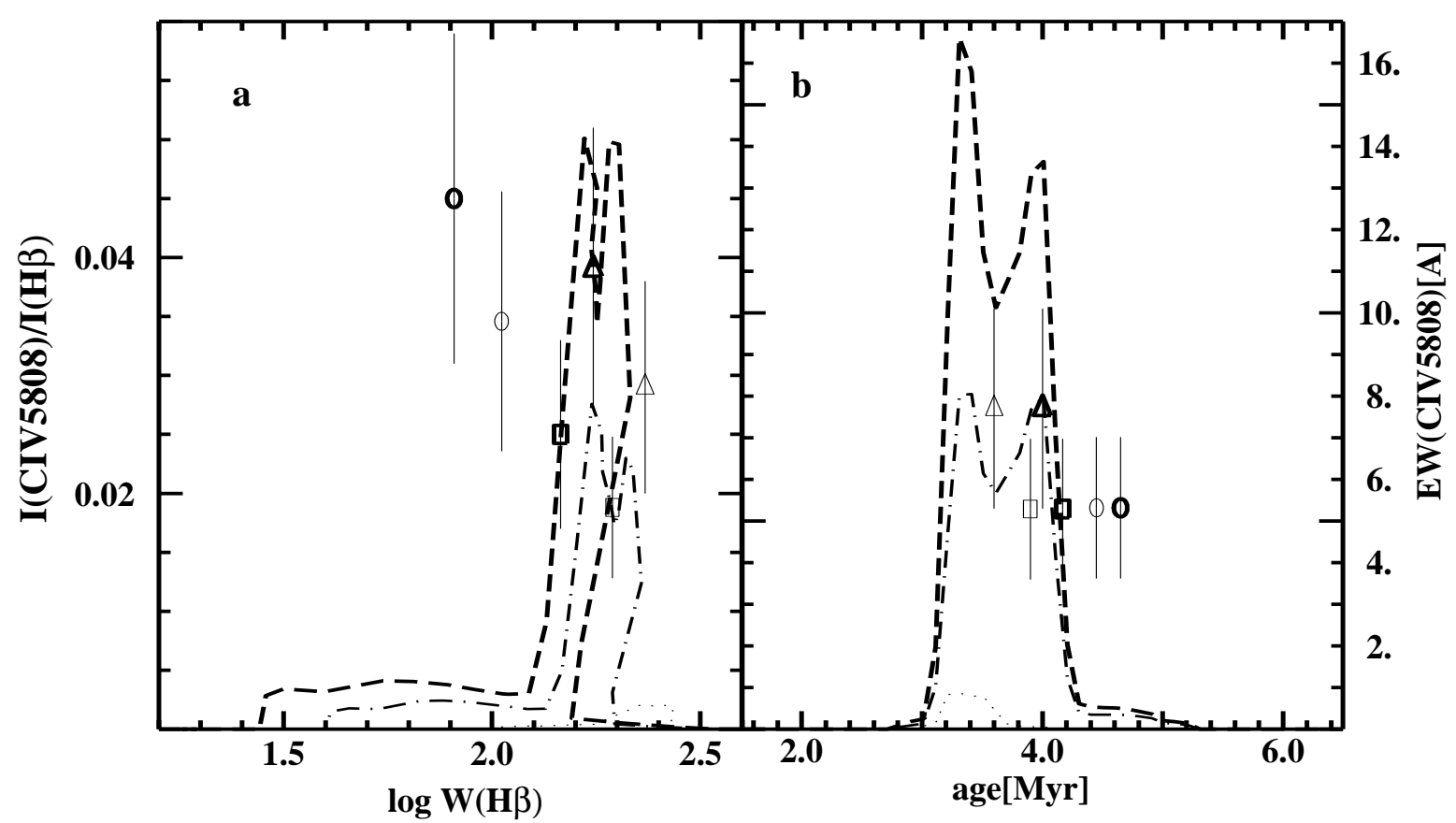




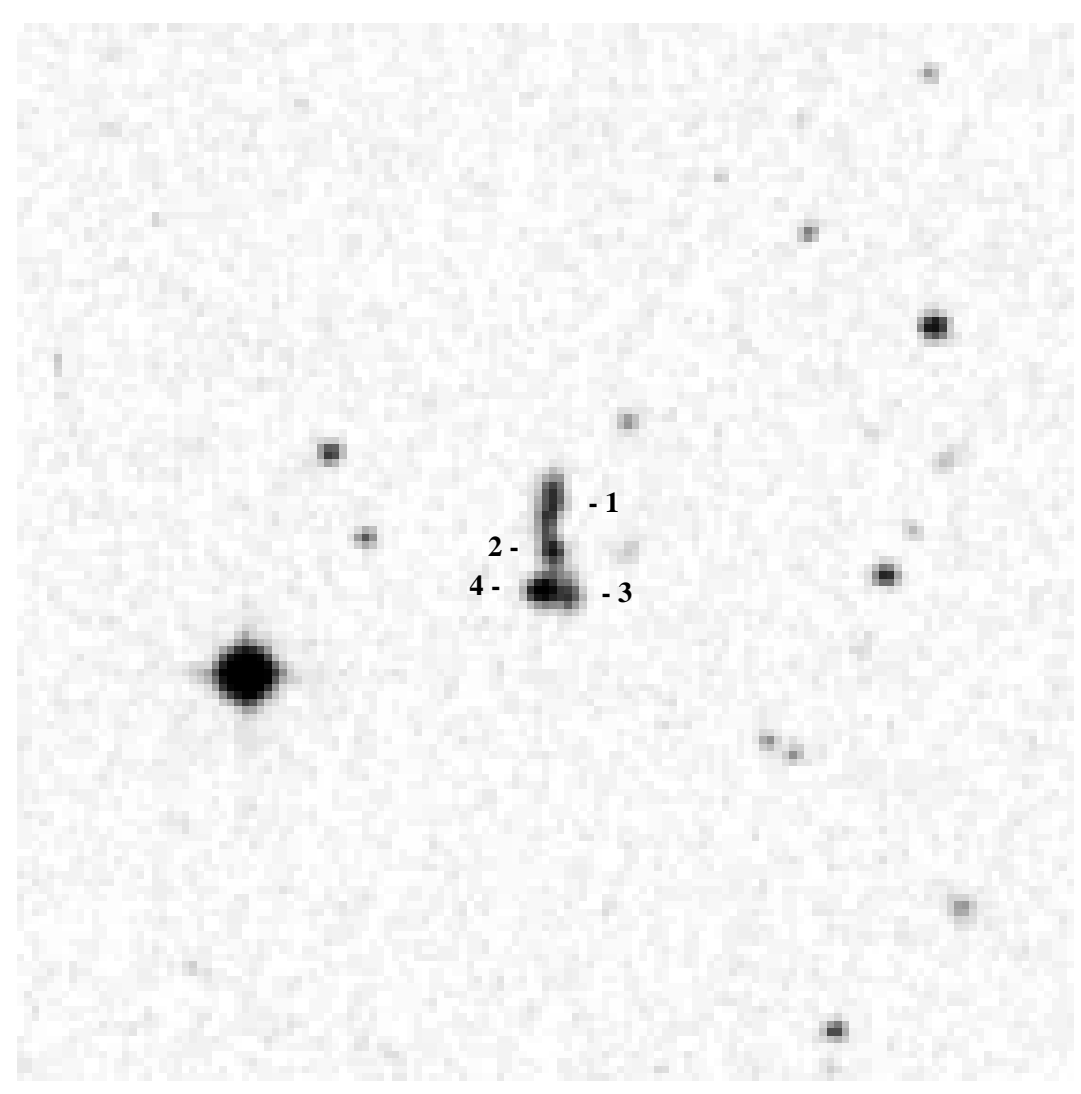

